Review

\title{
Patients at Risk for Peritoneal Surface Malignancy of Colorectal Cancer Origin: The Role of Second Look Laparotomy
}

\author{
Björn LDM Brücher1,7,8凶 , Alexander Stojadinovic'2,8, Anton J. Bilchik 3,8 , Mladjan Protic 4,8, Martin Daumer5,8, \\ Aviram Nissan ${ }^{6,8}$, Itzhak Avital7,8 \\ 1. Theodor-Billroth-Academy ${ }^{\circledR}$, Munich, Germany \\ 2. Department of Surgery, Walter Reed National Military Medical Center, Bethesda, MD, and the United States Military Cancer Institute, \\ Washington, D.C. USA \\ 3. John Wayne Cancer Institute, Santa Monica, CA, USA \\ 4. Clinic of Abdominal, Endocrine, and Transplantation Surgery, Clinical Center of Vojvodina, University of Novi-Sad, Medical Faculty, \\ Novi Sad, Serbia \\ 5. Sylvia Lawry Center for MS Research, Munich, Germany \\ 6. Department of Surgery, Hadassah University, Jerusalem, Israel \\ 7. Bon Secours Cancer Institute, Richmond, VA, USA \\ 8. INCORE = International Consortium of Research Excellence of the Theodor-Billroth-Academy®
}

$\varangle$ Corresponding author: Björn Brücher, MD, PhD, FRCS (Engl), FACS, Professor of Surgery, Theodor-Billroth-Academy, Josephsburgstrasse 6, 81673 Munich, Germany. Phone: +49 1792277 538. Email: b-bruecher@gmx.de

( ) Ivyspring International Publisher. This is an open-access article distributed under the terms of the Creative Commons License (http://creativecommons.org/ licenses/by-nc-nd/3.0/). Reproduction is permitted for personal, noncommercial use, provided that the article is in whole, unmodified, and properly cited.

Received: 2013.01.08; Accepted: 2013.02.13; Published: 2013.03.15

\begin{abstract}
Peritoneal surface malignancy (PSM) is a frequent occurrence in the natural history of colorectal cancer (CRC). Although significant advances have been made in screening of CRC, similar progress has yet to be made in the early detection of PSM of colorectal cancer origin. The fact that advanced CRC can be confined to the peritoneal surface without distant dissemination forms the basis for aggressive multi-modality therapy consisting of cytoreductive surgery (CRS) plus hyperthermic intra-peritoneal chemotherapy (HIPEC), and neoadjuvant and/or adjuvant systemic therapy. Reported overall survival with complete CRS+HIPEC exceeds that of systemic therapy alone for the treatment of PSM from CRC, underscoring the advantage of this multi-modality therapeutic approach. Patients with limited peritoneal disease from CRC can undergo complete cytoreduction, which is associated with the best reported outcomes. As early or limited peritoneal carcinomatosis is undetectable by conventional imaging modalities, second look laparotomy is an important means to identify disease in high-risk patients at a stage most amenable to complete cytoreduction. This review focuses on the identification of patients at risk for PSM from CRC and discusses the role of second look laparotomy.
\end{abstract}

Key words: Carcinomatosis, colorectal cancer, prospective, randomized, clinical trials, HIPEC, CRS, Oxaliplatin and Mitomycin

\section{Introduction}

Approximately 150,000 patients are diagnosed with colorectal cancer (CRC) in the US each year and one third of patients die annually from this disease [1]. Nearly 8,000 patients are diagnosed with syn- chronous peritoneal surface malignancy (PSM) each year [2]. Hence, PSM is a frequent manifestation in the natural history of colorectal cancer (CRC), and is associated with marked deterioration in quality of life 
(QOL) and limited survival. Despite advances in early detection of $C R C$, peritoneal disease spread continues to be a common mode of disease progression, as $8 \%$ of patients with CRC have synchronous peritoneal spread of disease at time of primary resection, and up to $25 \%$ of patients with recurrent colorectal cancer have disease confined to the peritoneal cavity [3].In about $30 \%$ of patients, PSM remains the primary reason for death in patients with CRC [4]. Confinement of disease to the peritoneal surface, in the absence of systemic metastasis, serves as the basis for surgical eradication of disease through aggressive multi-modality therapy consisting of cytoreductive surgery (CRS) plus hyperthermic intra-peritoneal chemotherapy (HIPEC). Overall survival in patients with PSM and CRC treated with systemic 5-Flourouracil (5-FU) alone is dismal, with mean survival of only 5 to 7 months [4, 5]. Overall survival is even worse $(\sim 3$ months) inpatients, who suffer from PSM due to CRC and have simultaneous bowel obstruction [6].

Aretrospective pooled analysis of over 2,000 study subjects enrolled in North Central Cancer Treatment Group (NCCTG) Phase III Trials N9741 and N9841 demonstrated a median survival of 12.7 months in patients with peritoneal spread of CRC [7]. Treatment-adjusted analysis showed that patients with PSM and CRC have worse survival compared to patients with advanced CRC and distant metastases without PSM ( $p=0.0006)$. Oncological outcome in patients with PSM of CRC origin treated by second line5-FU + Leucovorin + Oxaliplatin (FOLFOX) was not significantly improved. Progression-free survival was $\sim 6$ months. This is in contradistinction to reported median survival rates between 19 and 63 months in experienced centers using CRS +HIPEC to treat PSM of CRC origin, underscoring the advantage of this multi-modality therapeutic approach [8-11]. Although FOLFOX was found to be superior to Irinotecan +5 -FU/Leucovorin (IFL) and Irinotecan + Oxaliplatin (IROX) as first line therapy in the pooled analysis of the NCCTG trials by Franko et al., no survival benefit was apparent with second line use [7]. Systemic multi-drug chemotherapy has not altered the natural history of peritoneal carcinomatosis, as patients suffer disease progression and functional deterioration due to visceral obstruction, malignant ascites and cancer cachexia over a limited median survival [2].

The multi-modality therapy approach, using systemic chemotherapy plus aggressive CRS and HIPEC has shown clearly promising results. The randomized controlled trial (RCT) of Verwaal et al. demonstrated a statistically significant survival advantage for this therapeutic approach [9]. This was an
RCT comparing CRS + HIPEC versus 5-FU-based systemic chemotherapy, which demonstrated a significant OS benefit with median survival of 22 months versus 12 months and 2-year survival of $44 \%$ versus $22 \%$, respectively $[9,10]$. Other studies have shown that patients with peritoneal carcinomatosis from CRC treated with chemotherapy alone havea median survival of 5 to 13 months, whereas those treated with CRS + HIPEC for early peritoneal carcinomatosis from CRC have reported median survival in the range of $48-63$ months and 5-year survival of $\sim 50 \%$. Completeness of cytoreduction and limited disease are associated with longer survival, yet early peritoneal carcinomatosis is undetectable by conventional imaging.

A major problem in patients with PSM of CRC origin is that approximately $50 \%$ will have recurrence of disease after treatment $[12,13]$. These data could be generated only because some groups have performed so-called "second look laparotomy" to identify patients that could potentially benefit from second CRS + HIPEC, at a time when none of the patients had clinical or radiographic evidence of recurrent PSM [14-16]. The rationale for performing second look laparotomy is to identify PSM of CRC origin early in the natural history of the disease in patients at high risk of having disease recurrence, when tumor volume would be below an important threshold peritoneal cancer index (PCI) of 20, where the oncological impact of CRS + HIPEC conducted with curative intent is greatest [17].

The concept of second look operation in cancer treatment is more than 60years old, and was most likely established in 1948, when first described by Wangensteen [18-20]. Since that time various groups have reported the "second look approach" in a range of tumor entities for a number of oncological or surgical purposes: 1 . cancer staging; 2 . palliative treatment; and, 3. Multi-modality treatment (CRS + HIPEC) with curative intent. Second look laparotomy went through a renaissance for the treatment of PSM around the year 2000 [12-16, 18, 19, 21-37]. The early reports and history of second look operation have been described by Sugarbaker [12]. Although, second look laparotomy can successfully identify early PSM, its role and potential benefit has been specifically recommend to patients with high risk of developing peritoneal carcinomatosis [12].

There are a number of important facets to programmed second look laparotomy. First and foremost, surgical exploration and consecutive CRS and HIPEC are of major potential value if PSM can be identified early and complete cytoreduction can be achieved. It is anticipated that as data from different 
centers of excellence investigating second laparotomy accumulates important insights will be gained, particularly defining an optimal time point following operation for the primary CRC when a second laparotomy should be performed. Importantly, clinical and pathological parameters, and biomarkers will be identified that inform risk prediction models and clinical decision support tools to individualize risk stratification and treatment intervention. This precision medicine approach will in turn contribute to the development of novel targeted diagnostic and therapeutic approaches in the future.

\section{Evidence, rationale and suggested guide- lines for $2^{\text {nd }}$ look laparotomy}

Historical data suggests that patients presenting with perforated primary tumor, bleeding or obstructing lesions requiring emergent surgical intervention, those with regional nodal metastases, synchronous peritoneal carcinomatosis, and/or ovarian metastasesare at high risk of subsequent peritoneal carcinomatosis [12]. Esquivel and Sugarbaker investigated studied patients with PSM of appendiceal origin during a 12-year period [14]. Of 321 patients, 98 (31\%) underwent a second-look procedure (laparotomy, and CRS + HIPEC when indicated). The overall 5 -year survival rate in the 98/321 patients was $74 \%$ compared to $68 \%$ in $223 / 321$ patients. These data suggest that there is a sub-population of patients that can benefit from second look laparotomy and CRS + HIPEC. Those patients, who presented with symptomatic bowel obstruction or had a high peritoneal surface tumor burden, however, had significantly worse survival. As patients with large tumor burden $($ PCI >20) have questionable benefit from CRS + HIPEC, the role of second look operation following cytoreduction in these patients with high-volume disease is questionable [17].

In the study from France by Elias et al., 29 patients without clinical or radiographic evidence of PSM recurrence underwent second look operation 13 months after resection of the primary colon cancer [23]. More than $50 \%(16 / 29)$ of these patients had gross morphological evidence of PSM recurrence during second look laparotomy, corresponding to 10 patients with initial peritoneal carcinomatosis, three patients with synchronous ovarian metastases, and three patients with a perforated primary tumor. Eight out of these 16 patients $(50 \%)$ that underwent second look laparotomy remained disease-free, four had PSM relapse $(25 \%)$ and four $(25 \%)$ developed isolated visceral metastases after a median post-second look follow-up of 27 months. This study emphasizes the fact that PSM in CRC patients often does not have clini- cally- or radiographically-apparent signs until disease has reached an advanced stage with high tumor burden. Hence, the prevalence of the problem remains undefined. Importantly, at-risk patients are those having synchronous macroscopic PSM, synchronous ovarian metastases and perforated primary tumor. The French group published recently their experience with 41 patients over a 10-year time-period with a median follow up of 30 months [15] replicating their initial published results.

Maggiori et al. investigated 41 patients with PSM of CRC origin that underwent second look operation, who had no clinical or radiographic signs of peritoneal surface disease recurrence at the time of second look. Fifty-six percent $(23 / 41)$ of the asymptomatic patients with negative diagnostic imaging were found to have peritoneal surface malignancy which was treated with CRS + HIPEC at time of second look laparotomy, and 18 (44\%) underwent complete cytoreduction [16]. The reported Grade 3/4 morbidity rate was $9.7 \%$ with a 5 -year overall survival rate of $90 \%$, and a 5 -year disease-free survival rate of $44 \%$. Peritoneal recurrences occurred in 6 of the $23(26 \%)$ found to have PSM at second look laparotomy and treated with CRS+HIPEC, and in one of $18(6 \%)$ patients who had no evidence of PSM at time of second look exploration. This study emphasizes the fact that over $50 \%$ of at-risk patients with treated primary CRC will have gross manifestations of PSM at time of second look laparotomy, and that surgical intervention with CRS + HIPEC is associated with favorable oncological outcomes.

Sugarbaker emphasized clinical parameters that may support surgical decision making in at-risk patients and published suggested guidelines for second look laparotomy and CRS + HIPEC with curative intent $[12,13]$. To that end, he pointed out that the major focus must be complete cytoreduction (R0 resection, CC-0/1resection). He further argued that the limited extent of peritoneal surface tumor burden found at time of second look laparotomy increases likelihood of complete resection of grossly apparent disease, and optimizes the chances of favorably impacting prognosis. Patients considered to be at high-risk of having PSM at second look laparotomy are those: 1 . presenting with perforated primary CRC, or primary tumors causing bleeding or obstruction necessitating emergent surgical intervention; 2 : with regional nodal metastases; 3 . with synchronous peritoneal carcinomatosis; and/or, 4 . with ovarian metastases.

\section{Timing of second look laparotomy}

The aggressive approach of a so-called "programmed" second look operation is thought to be 
most clinically relevant in selected at-risk patients at a time when peritoneal tumor burden is low. The optimal timing of second look laparotomy following incident operation for the primary tumor remains to be defined. What we know thus far is that approximately $50 \%$ of patients at-risk for PSM will have PSM identified at time of second look laparotomy performed within approximately a year of primary operation $[14,16,23]$. This preliminary data based on incidence and extent of PSM at time of second exploration suggest that the preferred time point for performing second look laparotomy may be between 6 12 months of primary operation. In 2010 the NCI initiated a RCT (NCT01095523) for second look laparotomy to address the question: Does mandatory second look laparotomy with CRS + HIPEC prolong overall survival compared to the standard of care in patients who are at high risk for developing peritoneal carcinomatosis from CRC? [32]

\section{High risk factors, which may be respon- sible for early recurrence}

A number of risk factors have been identified that are associated with early recurrence of PSM in CRC. Positive peritoneal cytology, visible evidence of peritoneal carcinomatosis at time of initial operation, perforated primary tumor, and synchronous ovarian metastasis are among these risk factors. In addition, emergently treated bleeding or obstructed primary tumors or mucinous T3 stage or T4 stage tumors with adjacent organ involvement, as well as margin positive resection are high risk factors, which may be responsible for early recurrence of PSM of CRC origin [12].

Careful patient selection is an important component in the decision making process for second look laparotomy. Medical co-morbidities (heart, lung, kidney, low performance status), poor nutritional status, 3 or more liver metastases are relative contraindications for second look laparotomy. A recent study, however, suggested that morbidity is not significantly increased when performing hepatobiliary surgery during CRS and HIPEC [38]. Potential contraindications depending on severity of presentation include obesity, and acute abdomen with ileus [3]. Bucci et al. stated in 1994 that the serum tumor marker CEA (carcinoembryonic antigen) -directed second-look surgery is not a reliable or cost-effective approach [22]. An elevated serum CEA today prompts cross sectional and functional diagnostic imaging, which as discussed previously, is typically unrevealing for PSM of CRC origin. The above studies provide an evidence basis to forego imaging and proceed straight to second look surgery inpatients at high risk for PSM due to CRC.

Other factors that should be considered in selecting patients for second look laparotomy include tumor differentiation, and subtype (e.g. signet ring cell histology), synchronous versus metachronous PSM. It is well established that signet ring cell adenocarcinoma is a virulent form of gastrointestinal malignancy that is associated with a dismal prognosis; second look operation in patients with this tumor subtype is unlikely to be of significant benefit. Therefore, it is useful to determine the histopathological subtype of tumor in high risk patients by use of a precise immunohistochemical (IHC) approach [39]. A locally advanced primary tumor stage category reflects depth of tumor invasion with high likelihood of lymphatic dissemination, and it has been shown previously that more advanced $\mathrm{T}$-stage primary tumors such as Stage II and III T-4 tumors have $25 \%$ incidence of peritoneal spread as the only metastatic site of disease [40]. The Tumor-Node-Metastasis (TNM) staging classification is an evolving document based on advances in evidence-based medicine. In the future other disease-specific aspects may be taken into account as well; for example, recent morphometric analysis revealed that the peritoneal elastic lamina was a useful marker of level of tumor invasion, and powerful indicator of prognosis in CRC [41]. Interestingly it has been demonstrated that the angiogenic phenotype may differ between intestinal and diffuse type gastric cancer [42-44]. Translating these data to CRC signet ring cell adenocarcinoma, mucinous adenocarcinoma and perhaps metastatic phenotype may also be of value in risk stratification for PSM.

Lymphatic as well as vascular invasion and peri-neural infiltration are clinically relevant parameters amongst the various types of gastrointestinal cancers in terms of tumor biology and prognosis. The risk benefit ratio for second look operation in a patient with extensive lymphovascular invasion histopathologically would not favor this approach based on the low likelihood of benefit. Tumor heterogeneity remains a complex phenomenon that pertains to the primary tumor as well as the individual patient with advanced disease given the observed genotypic heterogeneity evident between the primary tumor and different metastatic sites [45].

Malignant ascites as a manifestation of peritoneal carcinomatosis is an adverse prognostic indicator. Sangisetty et al. recently showed that although patients with PSM and ascites may not be cured by a multi-modality approach, these patients may derive palliative benefit from laparoscopic heated intra-peritoneal chemotherapy [46]; randomized trials are not available and are needed [47]. The role of lap- 
aroscopic second look operation is unclear.

Multi-modality treatment approaches in both the neoadjuvant and adjuvant setting is an important part of the clinical pathway in gastrointestinal malignancy. Defining the value of adding systemic therapy after CRS + HIPEC, particularly after performing a second look laparotomy is an oncological challenge. Another goal of the future will be the assessment of treatment response, specifically selecting patients for multi-modality therapy based on reliable indicators of tumor biology [48]. However, careful consideration ought to be given to the fact that 35 years after the (only) experiment, which lead to the World Health Organization classification of tumor response to treatment, no additional studies have been conducted or proposed [49].

Targeted therapy directed by tumor specific testing at either the gene or protein expression is another basis for 'next generation' or precision cancer therapy. Despite rapid advances in our understanding of targeted therapy for GI cancers, the durable impact on cancer survival has been marginal overall. Targeted anti-angiogenic therapy, for instance, can also have unpredictable results and be a 'double-edged sword' for while vascular endothelial growth factor (VEGF) inhibitors reduce primary tumor growth, they may simultaneously promote tumor growth and metastatic spread through other mechanisms [50-52].

Intra-abdominal surgery leads to post-operative adhesions. The nature, extent, and functional impact of adhesions are highly variable. Minimal residual disease may reside within these adhesions; therefore, complete adhesiolysis is imperative during any kind of second look cancer operation and CRS + HIPEC with curative intent. The fact that tumor cells become sequestered in avascular intra-peritoneal adhesions explains partly the resistance to, and ineffectiveness of systemic chemotherapy alone for peritoneal carcinomatosis.

The presence of an anatomic barrier, the peritoneal-plasma partition, has enabled administration of high local concentrations of chemotherapy at the peritoneal surface, far in excess of systemically administered agents when drug delivery is intra-peritoneal as opposed to intravenous. High molecular weight agents such as Mitomycin C (334 Da), and Oxaliplatin (397 Da), for example, have favorable pharmacokinetic profiles (AUC, peritoneal fluid relative to plasma: Mitomycin C, 75:1; Oxaliplatin, 25:1) permitting dose-dense intra-peritoneal therapy over prolonged periods with rapid tissue concentration (in residual tumor deposits and peritoneum), but limited systemic absorption or toxicity. This particular therapeutic approach addresses the problem of systemic chemo- therapy resistance and, with its reduced systemic toxicity, provides distinct pharmacological advantage over systemic drug delivery. This makes completeness of cytoreduction imperative, which is conducted with the intent to eradicate macroscopic deposits of tumor and optimize the efficacy of hyperthermic chemotherapy in obliterating minimal residual disease. Optimal therapeutic synergy is achieved when intra-peritoneal heated chemotherapy is administered immediately following maximal cytoreduction, thereby minimizing trapping of viable peritoneal tumors cells in fibrin and post-operative adhesions, and maximizing kill of tumor cells shed during resection. Adhesions are lysed during cytoreduction to facilitate uniform distribution of heated chemotherapeutic perfusate, maximize direct contact of drug with residual peritoneal tumor cells, and harness the advantage of "thermo-chemotherapeutic" anti-tumor synergism.

Bristow et al. showed that using a hyaluronate-carboxymethylcellulose (HA-CMC) barrier for prevention of pelvic adhesion formation in women undergoing primary cytoreductive surgery with radical oophorectomy for locally advanced epithelial cancer is associated with a significant reduction in the extent and density of pelvic adhesion [53]. It might also be possible that, in the future, a patient who undergoes primary resection of a colon cancer and is found to have high-risk features, such as local PSM, that the operation is completed, HA-CMC applied to the viscera prior to abdominal closure, and the patient subsequently referred to a center of excellence in PSM for subsequent second look operation and cytoreductive surgery + HIPEC.

\section{New diagnostic and therapeutic options}

Knowles and Wu recently published a provocative report stating that Immuno-positron emission tomography (Immuno-PET) might provide a non-invasive approach, for obtaining target-specific information useful for titrating doses of radioimmunotherapy, for patient risk stratification and selection of targeted therapies, evaluation of therapeutic response, and for predicting adverse treatment effects [54]. Clinical implementation of Immuno-PET may indeed advance precision medicine. A validated gene expression signature that may predict recurrence in patients with early stage CRC was recently described [55].Levine et al. compared genetic profiles of patients with peritoneal metastases from colorectal and appendiceal primaries [56]. Efforts such as these and next generation sequencing of human tumor specimens may become an integral part of precision medicine, specifically clinical decision support systems that 
may risk stratify patients and individualize application of second look laparotomy and CRS + HIPEC as well as yield agent specific predictions of targeted systemic therapy response.

\section{Conclusions and future aspects}

Approximately $50 \%$ of patients who develop PSM from CRC may have curative multi-modality treatment including complete resection of all grossly apparent disease. Studies of patient with high-risk clinical and pathological features undergoing second look laparotomy have an incidence of PSM exceeding $50 \%$ following resection of the primary tumor. Unfortunately PSM cannot be detected pre-operatively in asymptomatic patients, as current diagnostic tests and/or standard imaging platforms lack sufficient sensitivity [2]. Factors identifying patients at high risk for peritoneal dissemination of CRC have been described and treatment guidelines suggested by Sugarbaker and the French Group [12, 23].Patients undergoing complete CRS + HIPEC (R0-resection)have a 2 -year survival of up to $60 \%$. Cytoreductive surgery, HIPEC and systemic chemotherapy are not competitive therapies - a fact emphasized in France based on broad a multi-modality therapeutic paradigm incorporated into the French Guidelines [23]. In Germany it will be integrated in to the treatment Guidelines as a therapeutic option in 2012. Patient selection is key to maximizing oncological benefit over aggregate risk of multiple treatment modalities (CRS + HIPEC + sys- temic therapy). Clinical decision support systems based on specific clinical, pathological, biomarker and patient data will ultimately facilitate risk stratification, further enable patient selection for second look laparotomy and individualize multi-modality therapy in patients with PSM in CRC. [57]

Combining different methodological approaches in the future will also be of great value. Immuno-PET antibody-based imaging will make possible detection of cell surface tumor biomarkers [54] and enhance the accuracy of multi-modality treatment response assessment. This diagnostic imaging platform will provide detailed determination of minimal residual disease as well as sub-clinical recurrence in patients with PSM CRC and fulfill an unmet need in functional diagnostic imaging. Novel diagnostic imaging platforms coupled with clinical decision support tools will make precision medicine possible for patients with PSM of CRC origin. This will efficiently and effectively direct efforts incorporating systematic second look surgery to maximize therapeutic benefit inpatients at risk for PSM, or in those asymptomatic patients with early PSM from CRC [26].

We suggest using an effective and comprehensible algorithm in treating patients suffering from primary colorectal carcinoma (Figure 1) as well as secondary colorectal carcinoma patients or those who had been selected already for $2^{\text {nd }}$ look operation (Figure 2).

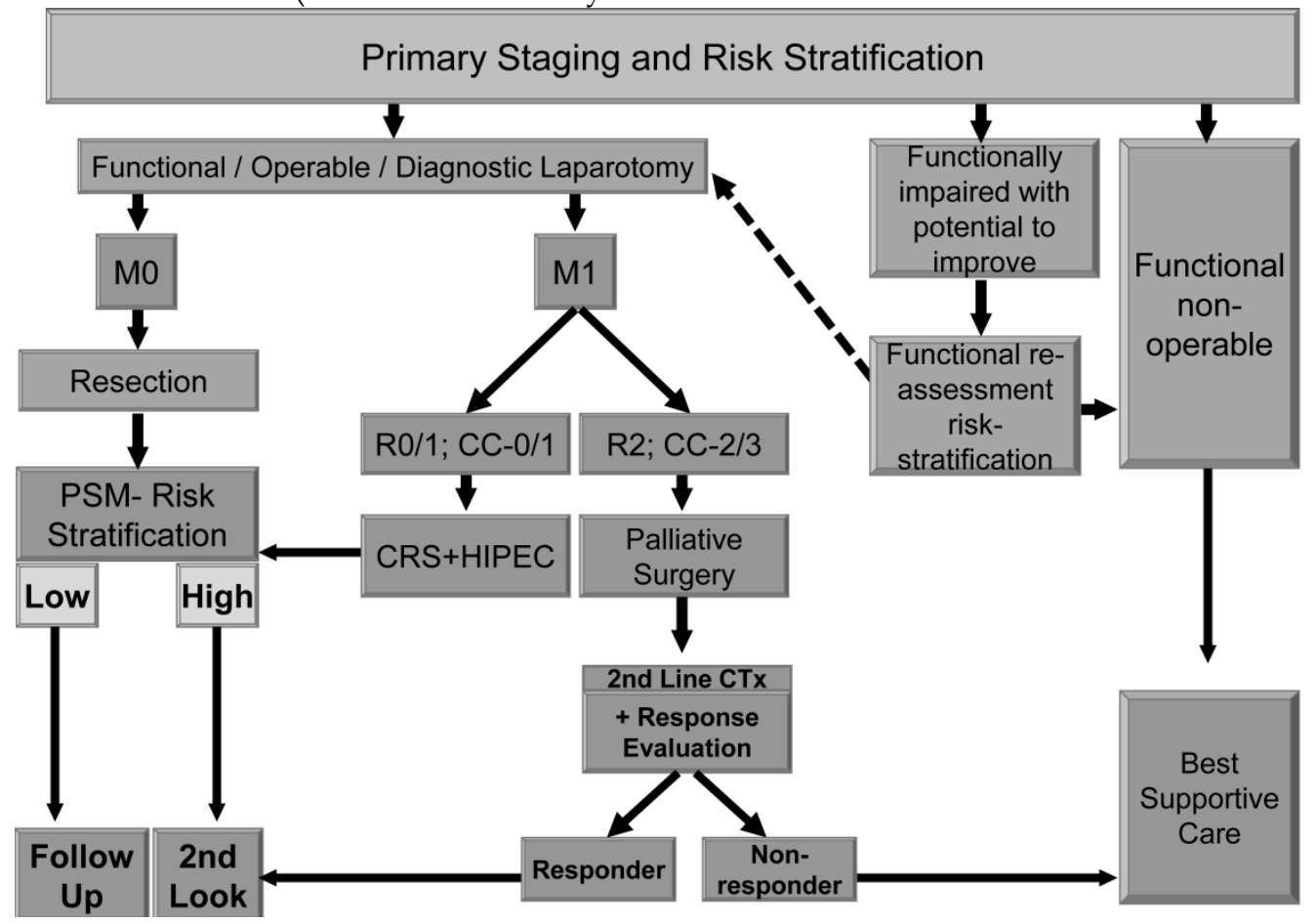

Figure I: CRC - PRIMARY DIAGNOSIS 


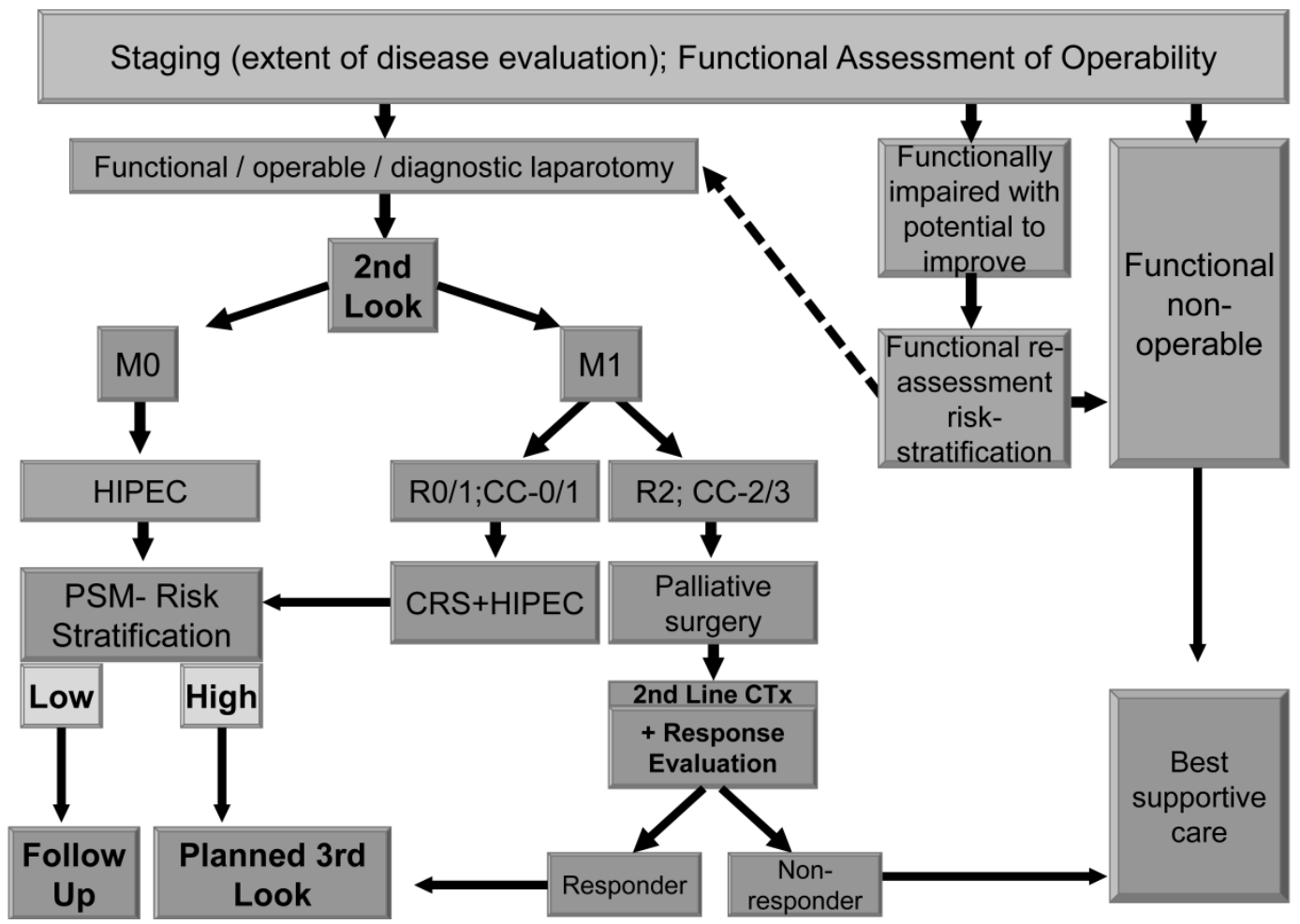

Figure 2: Resected CRC / Considered for 2nd Look in High Risk for PSM

\section{Abbreviations}

PSM: Peritoneal Surface Malignancy

CEA: carcinoembryonic antigen

CRC: Colorectal Cancer

CRS: Cytoreductive Surgery

5-FU: 5-Flourouracil

FOLFOX: 5-FU + Leucovorin + Oxaliplatin

HA-CMC: Hyaluronate-carboxymethylcellulose

HIPEC: Heated intra-peritoneal chemotherapy

IFL: Irinotecan + 5-FU/Leucovorin

IROX: Irinotecan + Oxaliplatin

NCCTG: North Central Cancer Treatment Group

PCI: Peritoneal Cancer Index

QOL: Quality of Life

RCT: Randomized Controlled Trial

VEGF: Vascular Endothelial Growth Factor

\section{Competing Interests}

The authors have declared that no competing interest exists.

\section{References}

1. Jemal A., et al. Cancer statistics, 2008. CA Cancer J Clin, 2008. 58(2): 71-96

2. Itzhak A, Brücher BLDM, Nissan A, Stojadinovic A: Randomized clinical trials for colorectal cancer peritoneal surface malignancy. Surg Clin North Am 2012; 21(4): 665-688.

3. Brücher BL, Piso P, Verwaal V, Esquivel J, Derraco M, Yonemura $\mathrm{Y}$, Gonzalez-Moreno S, Pelz J, Konigsriner A, Ströhlein M, Levine EA, Morris D, Bartlett D, Glehen O, Garofalo A, Nissan A. Peritoneal carci- nomatosis: cytoreductive surgery and HIPEC--overview and basics. Cancer Invest. 2012 Mar; 30(3):209-24

4. Jayne DG, Fook S, Loi C, Seow-Choen F: Peritoneal carcinomatosis from colorectal cancer. Br J Surgery 2002; 89(12):1545-50.

5. Chu DZ, Lang NP, Thompson C, et al. Pertoneal carcinomatosis in non gynecological malignancy: A prospective study of prognostic factors. Cancer 1989; 63: 364-36.

6. Blair SL, Chu DZ, Schwarz RE: Outcome of palliative operations for malignant bowel obstruction in patients with peritoneal carcinomatosis from nongynecological cancer. Ann Surg Oncol 2001; 8: 632-637.

7. Franko J, Shi Q, Goldman CD, Pockaj BA, Nelson GD, Goldberg RM, Pitot HC, Grothey A, Alberts SR, Sargent DJ: Treatment of colorectal peritoneal carcinomatpsis with systemic chemotherapy: a pooled analysis of North Central Cancer Treatment Group Phase III Trials N971 and N9841. J Clin Oncol 2012; 30: 263-276.

8. Verwaal V, Ruth S, Bree E, et al: Randomized trial of cytoreduction and hyperthermicintraperitoneal chemotherapy versus systemic chemotherapy and palliative surgery in patients with peritoneal carcinomatosis of colorectal cancer. J Clin Oncol 2003; 21:3737-3743.

9. Verwaal VJ, van Ruth S, Witkamp A, Boot H, van Slooten G, Zoetmulder FA: Long-term survival of peritoneal carcinomatosis of colorectal origin. Ann Surg Oncol. 2005 Jan; 12(1):65-71.

10. Elias D, GIlly F, Boutitie F, et al: Peritoneal carcinomatosis treated with surgery and perioperative intraperitoneal chemotherapy: retrospective analysis of 523 patients from a multicentric French study. J Clin Oncol 2010; 28(1): 63-68.

11. Glehen O, Kwiatkowski F, Sugarbaker P, et al: Cytoreductive surgery combined with perioperative intraperitoneal chemotherapy for the management of peritoneal carcinomatosis from colorectal cancer: a multi-institutional study. J Clin Oncol 2004; 22(16): 3284-3.

12. Sugarbaker PH: Revised guidelines for second-look surgery in patients with colon and rectal cancer. Clin Transl Oncol. 2010 Sep;12(9):621-8.

13. Sugarbaker P: Second-look surgery for colorectal cancer: revised selection factors and new treatment options for greater success. Int J Surg Oncol. 2011;2011:915078.

14. Esquivel J,Sugarbaker PH: Second-look surgery in patients with peritoneal dissemination from appendiceal malignancy: analysis of prognostic factors in 98 patients. Ann Surg. 2001 Aug;234(2):198-205.

15. Elias D, Honoré C, Dumont F, et al: Results of systematic second-look surgery plus HIPEC in asymptomatic patients presenting a high risk of 
developing colorectal peritoneal carcinomatosis. Ann Surg. 2011 Aug; 254(2):289-93.

16. Maggiori L,Elias D: Curative treatment of colorectal peritoneal carcinomatosis: current status and future trends. Eur J Surg Oncol. 2010 Jul;36(7):599-603.

17. Jacquet $P$, Sugarbaker $P H$ : Current methodologies for clinical assessment of patients with peritoneal carcinomatosis. J Exp Clin Cancer Res 1996; 15: 49-58.

18. Wangensteen $\mathrm{OH}$ : Cancer of the colon and rectum; with special reference to earlier recognition of alimentary tract malignancy; secondary delayed re-entry of the abdomen in patients exhibiting lymph node involvement; subtotal primary excision of the colon; operation in obstruction. Wis Med J 1949; 48: 591-597

19. Wangensteen $\mathrm{OH}$, Lewis FJ, Tongen LA: The second look in cancer surgery: a patient with colic cancer and involve lymph nodes negative on the sixth look. J Lancet 1951; 71(8): 303-307.

20. Griffen WO Jr, Gilbertsen VA,Wangensteen OH: The second look opeation for abdominal malignancies 1948-1963. Natl Cancer Inst Monogr 1964 15: 267-276

21. Bleday R, Steele GJr: Second-look surgery for recurrent colorectal carcinoma: is it worthwhile? Semin Surg Oncol. 1991;7(3):171-6.

22. Bucci L, Benassai G, Santoro GA: Second look in colorectal surgery. Dis Colon Rectum. 1994 Feb;37(2 Suppl):S123-6.

23. Elias D, Goéré D, DiPietrantonio D, Boige V, Malka D, Kohneh-Shahri N, Dromain C, Ducreux M: Results of systematic second-look surgery in patients at high risk of developing colorectal peritoneal carcinomatosis. Ann Surg. 2008 Mar;247(3):445-50.

24. Gilbertsen VA, Wangensteen OH: A summary of thirteen years' experience with the second look program. Surg Gynecol Obstet 1962; 114: 438-442.

25. Grotz RL, Pemberton JH: Second look surgery--a shell game? Am J Gastroenterol. 1992 Jun;87(6):804-5.

26. Lefevre JH, Elias DM: Cytoreductive surgery plus intraperitonealchemohyperthermia in patients with colorectal cancer at high risk for local-regional recurrence. Cancer J. 2009;15(3):200-3.

27. Martin EW Jr, James KK, Hurtubise PE, Catalano P, Minton JP: The use of CEA as a nearly indicator for gastrointestinal tumor recurrence and second-look procedures. Cancer. 1977 Feb;39(2):440-6.

28. Martin EWJr, Carey LC: Second-look surgery for colorectal cancer. The second time around. Ann Surg. 1991 Sep;214(3):321-5.

29. Minton JP, Hoehn JL, Gerber DM, Horsley JS, Connolly DP, Salwan F, Fletcher WS, Cruz ABJr, Gatchell FG, Oviedo M, et al: Results of a 400-patient carcinoembryonic antigen second-look colorectal cancer study. Cancer. 1985 Mar 15;55(6):1284-90.

30. Osteen RT, Guyton S, Steele G Jr, Wilson RE: Maligant intestinal obstruction. Surgery 1980; 87: 611-615.

31. Portilla AG, Sugarbaker PH, Chang D: Second-look surgery after cytoreduction and intraperitoneal chemotherapy for peritoneal carcinomatosis from colorectal cancer: analysis of prognostic features. WorldJSurg. 1999 Jan;23(1):23-9.

32. Ripley RT, Davis JL, Kemp CD, Steinberg SM, Toomey MA, Avital I: Prospective randomized trial evaluating mandatory second look surgery with HIPEC and CRS vs. standard of care in patients at high risk of developing colorectal peritoneal metastases. Trials. 2010 May 25;11:62.

33. Rittgers RA, Steele G Jr, Zamcheck N, Loewenstein MS, Sugarbaker PH, Mayer RJ, Lokich JJ, Maltz J, Wilso RE: Transient carcinoembryonic antigen (CEA) elevations following resection of colorectal cancer: a limitation in the use of serial CEA levels as an indicator for second-look surgery. J Natl Cancer Inst. 1978 Aug;61(2):315-8.

34. Santoro BT, Griffen WO Jr, Wangensteen C: The second look procedure in the management of ovarian malignancies and pseudomyxomaperitoei. Surgery 1961; 50: 354-358.

35. Spears H, Petrelli NJ, Herrera L, MItelman A: Treatment of bowel obstruction after operaion for colorectal carcinoma. Am J Surg 1998; 155: 383-386.

36. Walsh HPJ, Schofield PF: Is laparotomy for small bowel obstruction justified in patients with previously treated malignanvy? Br J Surg 1984; 71: 933-935.

37. Zivanovic O, Barakat RR, Sabbatini PJ, Brown CL, Konner JA, Aghajanian CA, Abu-Rustum NR, Levine DA: Prognostic factors for patients with stage IV epithelial ovarian cancer receiving intraperitoneal chemotherapy after second-look assessment: results of long-term follow-up. Cancer. 2008 Jun 15;112(12):2690-7.

38. Glockzin G, Renner P, Popp FC, Dahlke MH, von Breitenbuch P, Schlitt I, Piso P: Hepatobiliary procedures in patients undergoing cytoreductive surgery and hyperthermic intraperitoneal chemotherapy. Ann Surg Oncol 2011; 18(4): 1052-1059.
39. Takahashi S, Kuroiwa G, Hirayama M, Tobioka H: [Advanced poorly differentiated adenocarcinoma of the colon with micropapillarycarcinoma components except specific cytokeratin expressions, report of a case]. NihonShokakibyoGakkaiZasshi. 2011 Dec;108(12):2016-22.

40. Hompes D, Tiek J, Wolthuis A, Fieuws S, Penninckx F, Van Cutsem E, D'Hoore A. HIPEC in T4a colon cancer: a defendable treatment to improve oncologic outcome? Ann Oncol. 2012; [Epub ahead of print].

41. Kojima M, Nakajima K, Ishii G, Saito N, Ochiai A: Peritoneal elastic laminal invasion of colorectal cancer: the diagnostic utility and clinicopathologic relationship. AmJSurgPathol. 2010 Sep;34(9):1351-60.

42. Takahashi Y, Clearly KR, Mai M, Kitadai Y, Bucana CD, et al. Significance of vessel count and vascular endothelial growth factor and its receptor (KDR) in intestinal type gastric cancer. Clin Cancer Res 1996;2: $1679-1684$.

43. Yamamoto S, Yasui W, Kitadai Y, Yokozaki H, Haruma K, et al. Expression of vascular endothelial growth factors in human gastric carcinomas. Pathology International 1998;48: 499-506.

44. Kitadai Y. Angiogenesis and Lymphangiogenesis of gastric cancer. J Oncol 2010;10: 468725.

45. Longo DL: Tumor heterogeneity and personalized medicine. NEJM 2012; 366(10): 956-957.

46. Sangisetty SL, Miner TJ: Malignant ascites: A review of prognostic factors, pathophysiology and therapeutic measures. World J GastrointestSurg. 2012 Apr 27;4(4):87-95.

47. Shapiro JF, Chase JL, Wolff RA, et al. Modern systemic chemotherapy in surgically unresectable neoplasms of appendiceal origin: a single-institution experience. Cancer 2010;116:316-322.

48. Brücher BLDM: Assessment of histopathological response assessment by residual tumor cell quantificatin in esophageal squamous cell carcinoma after neoadjuvant therapy: What for? American Journal of Hematology / Oncology AJSOR 2006; 5(10): 619-624.

49. Brücher BL, Bilchik A, Nissan A, Avital I, Stojadinovic A: Tumor response criteria: are they appropriate? Future Oncol. 2012 Aug;8(8):903-6.

50. Ebos JM, Lee CR, Cruz-Munoz W, Bjarnason GA, Christensen JG, Kerbel RS: Accelerated metastasis after short-term treatment with a potent inhibitor of tumor angiogenesis. Cancer Cell 2009;15:232-239.

51. Loges S, Mazzone M, Hohensinner P, Carmeliet P. Silencing or fueling metastasis with VEGF inhibitors: antiangiogenesis revisited. Cancer Cell 2009;15:167-170.

52. Paez-Ribes M, Allen E, Hudock J, Takeda T, Okuyama H, Vinalis F, Inoue M, Bergers G, Hanahan D, Casanovas O: Antiangiogenic therapy elicits malignant progression of tumors to increased local invasion and distant metastasis. Cancer Cell 2009;15:220-231.

53. Bristow RE, Montz FJ: Prevention of adhesion formation after radical oophorectomy using a sodium hyaluronate-carboxymethylcellulose (HA-CMC) barrier. Gynecol Oncol. 2005 Nov;99(2):301-8.

54. Knowles SM, Wu AM: Advances in Immuno-Positron Emission Tomography: Antibodies for Molecular Imaging in oncology. J Clin Oncol 2012; epub.

55. Gray RG, Quirke P, Handley K, et al. Validation study of a quantitative multigene reverse transcriptase-polymerase chain reaction assay for assessment of recurrence risk in patients with stage II colon cancer. J Clin Oncol 2011;29:4611-4619.

56. Levine EA, Blazer DG 3rd, Kim MK, Shen P, Stewart JH 4th, Guy C, Hsu DS: Gene expression profiling of peritoneal metastases from appendiceal and colon cancer demonstrates unique biologic signatures and predicts patient outcomes. Jam Coll Surg. 2012 Apr;214(4):599-606.

57. Stojadinovic A, Bilchik A, Smith D, Eberhardt JS, Ward EB, Nissan A, Johnson EK, Protic M, Peoples GE, Avital I, Steele SR: ClinicalDecisionSupportandIndividualizedPredictionofSurvivalinColonCancer: Bayesian Belief Network Model. Ann Surg Oncol. 2012; [Epub ahead of print]. 